\title{
Towards an integrated approach to tailings management
}

A. Chryss CSIRO Process Science and Engineering, Australia

A.B. Fourie School of Civil and Resource Engineering, The University of Western Australia, Australia

A. Mönch CSIRO Process Science and Engineering, Australia

D. Nairn CSIRO Process Science and Engineering, Australia

K.D. Seddon ATC Williams Pty Ltd, Australia

\section{Abstract}

Tailings management aims to dispose of tailings at the minimum cost consistent with meeting or exceeding imposed standards, including legislative obligations. This simple business objective requires managing the tailings storage facility (TSF) risks associated with surface water, groundwater, dust, sustainable closure and potential catastrophic failure. The individual nature of every ore body and specific site topography ensures that no 'one size fits all'.

It has been suggested that paste and thickened tailings (P\&TT) provides the ability to tailor the production and transportation of tailings to suit the deposition site, rather than the traditional approach of accepting that the deposition site has to accommodate tailings of high variability. While the traditional approach can be made to work, it is directly linked to the catastrophic failures of tailings impoundments, where large volumes of decant water have triggered failures due to overtopping, piping or slope instability, or saturated tailings have simply liquefied.

$P \& T T$ offers an ability to mitigate these risks and when implemented into new projects with variable tailings streams there are significant benefits to be realised. The paper identifies the inter-dependencies between the various tailings disposal processes, such as thickening, pumping and deposition. It demonstrates that there are benefits in taking a more integrated approach to the design and operation of tailings disposal facilities.

A collaborative industry project to tackle this challenge is described.

\section{Introduction}

The scarcity of water supply in mined arid regions and the environmental constraints placed upon surface tailings disposal requires, more than ever, that tailings management remains a high priority in any mineral processing or mining operation (DITT, 2007). The move towards higher solids concentrations in tailings streams means that, as with other process streams, they are no longer simple heterogeneous suspensions, like sand in water, which can be readily prepared, pumped and distributed throughout the impoundment. Instead, the materials are non-Newtonian hybrid suspensions, often thixotropic, that can be difficult to prepare, transport, distribute and consolidate (Nguyen and Boger, 1998). Materials are difficult to thicken to these concentrations, pumping has special considerations and the properties of the slurry as it passes from the thickeners to a disposal site may change considerably due to the shear forces inherent within the transportation processes. Once there, distribution of this now ill-defined material is complicated by the need to predict how thickened materials will self-distribute or whether new distribution techniques are required. Optimisation of each process is not simple, as the complexity of the non-Newtonian interactions increase as the solids concentrations approach their maximum packing conditions.

New technologies and techniques for tailings disposal have been proposed and developed in the last two decades (Jewell and Fourie, 2006). Many remain untested in the field, or have not made it to the open literature and need comparison to competing or traditional means. Greater understanding of the 
underlying principles and quantification of the outcomes will give the means to integrate the various components into a managed tailings scheme. The integration of the study of tailings processes will allow the prediction of downstream outcomes from changes in upstream conditions, and better informed design decisions. Conversely, upstream conditions may be designed or changed to ensure that the ultimate storage of the material is satisfactorily achieved. What follows is a description of some key areas that need to be addressed to allow the planned integration of the understanding and management of tailings processes.

\section{The need for an integrated approach}

Despite the improvements made in the last few decades and the achievements of experts in the field, increasing demands are being placed on practitioners involved in tailings management. This arises from several directions. More stringent legislation demanding specific outcomes from TSFs and mining in potentially more arid or tropical regions are some of the drivers. The need to maximise water recovery in arid climates is particularly pressing. The mining of lower grade and/or complex ores will produce greater quantities of tailings per tonne of product as well as increasing the fines content of the tailings. New technologies and strategies will need to be implemented in tailings management to ensure a continuing social licence to operate and to minimise costs over the life of the TSF.

The challenge of designing a generic tailings management solution is a complex one, as different ore types, bodies, locations and climates all combine to produce a diverse array of tailings. A 'one size fits all' solution is clearly not feasible but enough similarities may be found across the majority of tailings types to establish the basic principles that need to be considered and these can be refined for a specific application. One possible breakdown of these issues is as follows:

- water management and thickening

- transport of tailings

- stack configuration and growth

- TSF stability

- environmental concerns

- operating versus capital costs.

The boundaries drawn here are somewhat arbitrary, as water management impacts on environmental concerns, just as stack growth and TSF stability are inter-related. This further highlights that any advanced management solution must recognise the inter-connected nature of the tailings process. The tailings process must be designed and optimised as an integrated system.

\section{$3 \quad$ A process strategy}

What is the best approach to produce a robust, adaptable integrated tailings management solution? If the aim of tailings management is the establishment of a remediable TSF containing a predictable quantity of solids, then it would be intuitive that all operations within the tailings circuit should be optimised to achieve that end, and design should start from the tailings beach and work upstream. This is an approach that has been mentioned in tailings literature before (Boger, 2011), but has not been developed to a high degree. This simple view is complicated by the other functions of the tailings circuit such as water storage and reagent recovery, as well as the necessity of minimising footprint and cost. Thinking of the TSF as having a hierarchy of needs in this way is unhelpful since it is an inter-connected process where upstream changes produce multiple effects on depositing and distribution, as well as pumping, pipeline pressure drops, deposition strategy and even final closure options.

A more useful analogy can be taken from the manufacturing sector. If tailings management is viewed not as the disposal of an unwanted material in some optimal manner but as a process to manufacture a product, 
in this case a legislation-compliant, remediable tailings storage facility (a 'Walk away TSF'), then the priorities can be ordered and acted upon. In this scenario, the product has no commercial value but still needs to be carefully manufactured at minimal cost, while meeting certain performance criteria. The performance criteria derive from legislative and environmental drivers and specify the TSFs stability, drainage, trafficability and ease of remediation. For example, the Energy Resources Conservation Board of Alberta, Directive 074 (ERCB, 2009) sets definite performance criteria on undrained shear strength in a TSF as a function of time. To complete the analogy, the raw materials in the manufacturing process are tailings, water and flocculant and the unit operations are thickening and/or filtering, transporting and spreading or deposition.

To optimise the whole process, robust models of the component unit operations are needed. Some of these areas have been the subject of recent research and are at a well developed stage of understanding. The essential tools required for describing the tailings process would include:

- An understanding of rheological behaviour as it changes with time and processing. As the tailings solids pass through the process from thickening and pumping to deposition, the slurry is changing in concentration and aggregation state, and at times also in chemical composition and activity. The modelling of the various unit processes will require the ability to understand this behaviour and will necessitate quantifying the more complex aspects of rheology and not simply the static yield stress. Evaluating tailings slurries according to rheological and settling behaviour will allow slurries to be understood as a continuum and not arbitrarily classified as just 'paste' or 'thickened tailings'. Comprehending the rheological behaviour is integral to the points below.

- A descriptive model of thickener behaviour. This entails the modelling of a thickener's behaviour that produces outputs not for a single operating point but allows for thickener performance variability and provides a distribution of output values of throughput, underflow density and rheology. As discussed in a later example, these variations impact strongly on downstream operations such as pumping and deposition in the TSF.

- Modelling of pipeline (or gravity channel) flow behaviour of tailings in laminar, transitional and turbulent flows, allowing for settling of coarse fractions. The complex and stratified flows that can occur from pumping non-Newtonian and heterogeneous slurries through pipelines several kilometres in length, make design and operation difficult. In the context of this proposed strategy, upstream variations will be better dealt with if the flow regime is correctly described, as will be illustrated in the examples below.

- A better understanding of undrained strength of deposited tailings. Consolidation due to evaporative drying and seepage from homogeneous material has been modelled but does not necessarily reflect the true circumstances occurring on the TSF. The various strata that exist after each layer of tailings is deposited lose water at different rates, and the resulting inhomogeneous material has complex behaviour. Quantifying water release rates is also necessary to accurately predict the best deposition conditions for water recovery (Vietti et al., 2010; Seddon and Dillon, 2009). An understanding of this behaviour is essential to meeting the TSF performance criteria.

- A tailings stack growth model based on sound principles. Of the three main models used to predict beach slope (Simms et al., 2011) none is strongly grounded in theory as they are based on Newtonian fluid assumptions. Consequently, they lack the ability to be readily adapted to new slurry and process conditions without extensive large scale testing due to the non-Newtonian behaviour of thickened tailings. Modelling the flows of discharged tailings on the stack and incorporating upstream process variations will be a means of achieving this end, ideally creating a robust means of predicting beach slope across a wide range of mineral types and without the need for large quantities of representative tailings.

It is relatively easy to identify an 'optimum/ minimum cost' solution for each individual component of the tailings process. It is far harder to identify a process optimum. The integration of these models will allow for 
the evaluation and validation of various TSF flowsheet scenarios around a range of key performance constraints such as local environmental requirements, water recovery targets, flocculent optimisation, operating and capital costs. Application of dynamic techno-economic modelling would provide the decision making basis for efficient deployment of resources and could be used to identify likely operational risks and bottlenecks in the tailings management process. The development of a robust technical and economic basis for implementing leading practice in tailing management is critical to advancing this increasingly important area of mineral processing operations. Not only can the failure or poor performance of a TSF have a severe impact on the corporate bottom line, it can also significantly impact on a company's reputation and its social licence to operate (DITT, 2007). The reduced risk of a major tailings incident will more than offset the cost of developing more comprehensive tailings management systems.

The balance between initial capital expenditure, operating and deferred rehabilitation costs is changing as longer term economic perspectives are increasingly incorporated into the net present value calculations of proposed and ongoing mining operations. Consideration of closure, rehabilitation and post-closure management during TSF development will ensure optimal balance of lifetime economic, environmental and societal performance.

\section{Process examples}

The following two examples illustrate the need for an integrated approach to tailings processes. Neither was conducted using the method described above in its entirety but contain key elements of it.

\section{1 Thickener improvements also improve TSF performance}

Around 2004, the AngloGold Ashanti Sunrise Dam mine in Western Australia was experiencing trouble in achieving the design beach slope required for its centrally thickened discharge (CTD) tailings facility. The cause was traced upstream to the thickener which was performing poorly, with very high flocculant consumption (and hence occasional 'doughnut' formation) and low underflow density ( $55 \mathrm{wt} \%$ ). After redesign of the feedwell via CFD modelling, flocculation efficiency improved significantly, allowing the flocculant dosage to be reduced by $\sim 0.1 \mathrm{~kg}$ per tonne. As a consequence of the improved flocculation efficiency, the underflow solids increased from $55 \mathrm{wt} \%$ to $60-66 \mathrm{wt} \%$. This higher solids concentration produced a more robust yield stress, i.e. where extended pipe flow did not result in a significant change in the yield stress and other rheological parameters. The result was that the beach slope increased to $2 \%$, substantially enhancing the TSF storage capacity. Added benefits were the amount of water sent to the TSF was reduced by $\sim 25 \%$, providing more immediate process water as well as reduced cyanide losses.

The concentration and yield stress profiles along the Sunrise Dam tailings circuit are represented schematically in Figure 1 and Figure 2. Figure 1 shows the low concentration, over-flocculated slurry before the feedwell modification, with Figure 2 showing the improved performance after the feedwell modification. There is also a benefit to pumping costs in the reduction of water used, but the effect on specific energy consumption (SEC) of the higher viscosity slurry being pumped over the $3 \mathrm{~km}$ tailings pipeline was not examined. This example shows that improving thickener performance can also result in significant downstream TSF improvement. 


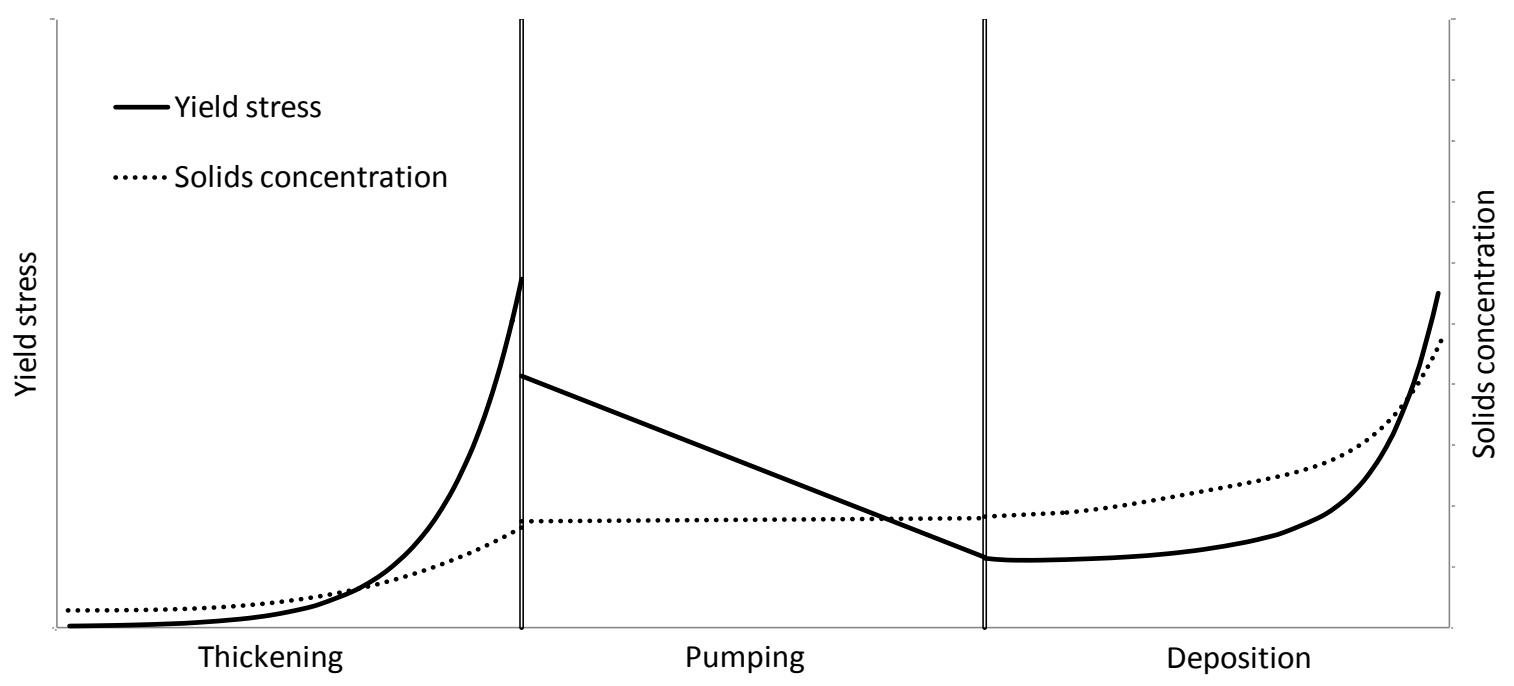

Figure 1 Schematic of Sunrise Dam suspension profiles before feedwell changes, showing excessive flocculation during thickening and no beach formation with segregation during deposition

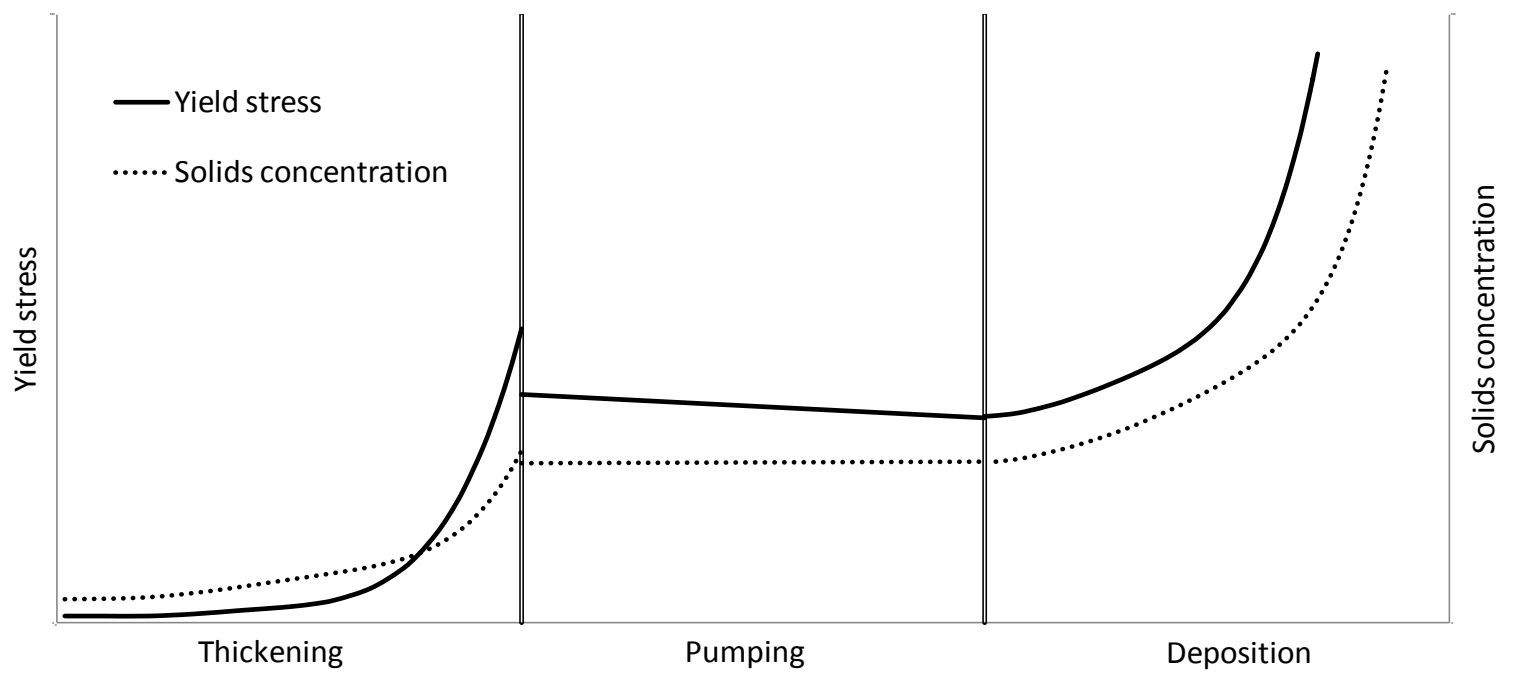

Figure 2 Schematic of Sunrise Dam suspension profiles after feedwell changes, showing improved flocculation during thickening and improved beaching, with little segregation during deposition

\section{2 Pipeline control and TSF performance}

A less straightforward example with complex interactions is as follows. Treating pipeline flows as heterogeneous materials is a simplification that can lead to process difficulties. Taking the case of a coarse material being transported in a non-Newtonian carrier (consisting of water and fine material), the usual pressure drop/velocity function is approximately $d P=f\left(V^{2}\right)$. This is not necessarily the case when a sliding bed of solids is formed and an almost flat response arises (Figure 3). This situation is difficult to control using centrifugal pumps, but even in this case where positive displacement pumps were used, control remained difficult, as it was based on the expected pressure relationship, which appeared to be unresponsive. Should there be a reduction in flow rate from, for example, the thickener or a drop in slurry viscosity due to typical process changes, then the bed could even increase in depth, resulting in an increase in pressure drop with reduced flow rate - the opposite to the expected behaviour. Should the operators then apply the standard response to rising pressures and dilute the slurry then the carrier suspension will 
be reduced in viscosity and lose its capacity to drag the coarser solid bed along, potentially causing the line to block. Should the operator be fortunate enough to avoid blocking the line by this action the higher flow rate of low concentration tailings will erode or wash out the tailings beach. The solution in this case is (a) to understand the flow mechanism and (b) if in doubt increase the slurry concentration, not dilute it. This illustrates the critical need to understand the interdependence that exists between the thickening, pumping and deposition unit operations to avoid sub-optimal tailings disposal.

Pipeline transport of thickened tailings is often complicated due to the economic considerations of pumping over long distances. The SEC of thickened tailings has been shown to be a minimum for transitional flows (Pullum, 2007) for a range of slurry types. This flow regime can be hard to maintain if the rheology is changing as the slurry moves through the pipeline (which can be exacerbated by long pipeline lengths) or changing with variations in process conditions. Robust knowledge and on-line measurement of the slurry rheology is required to achieve the necessary control.

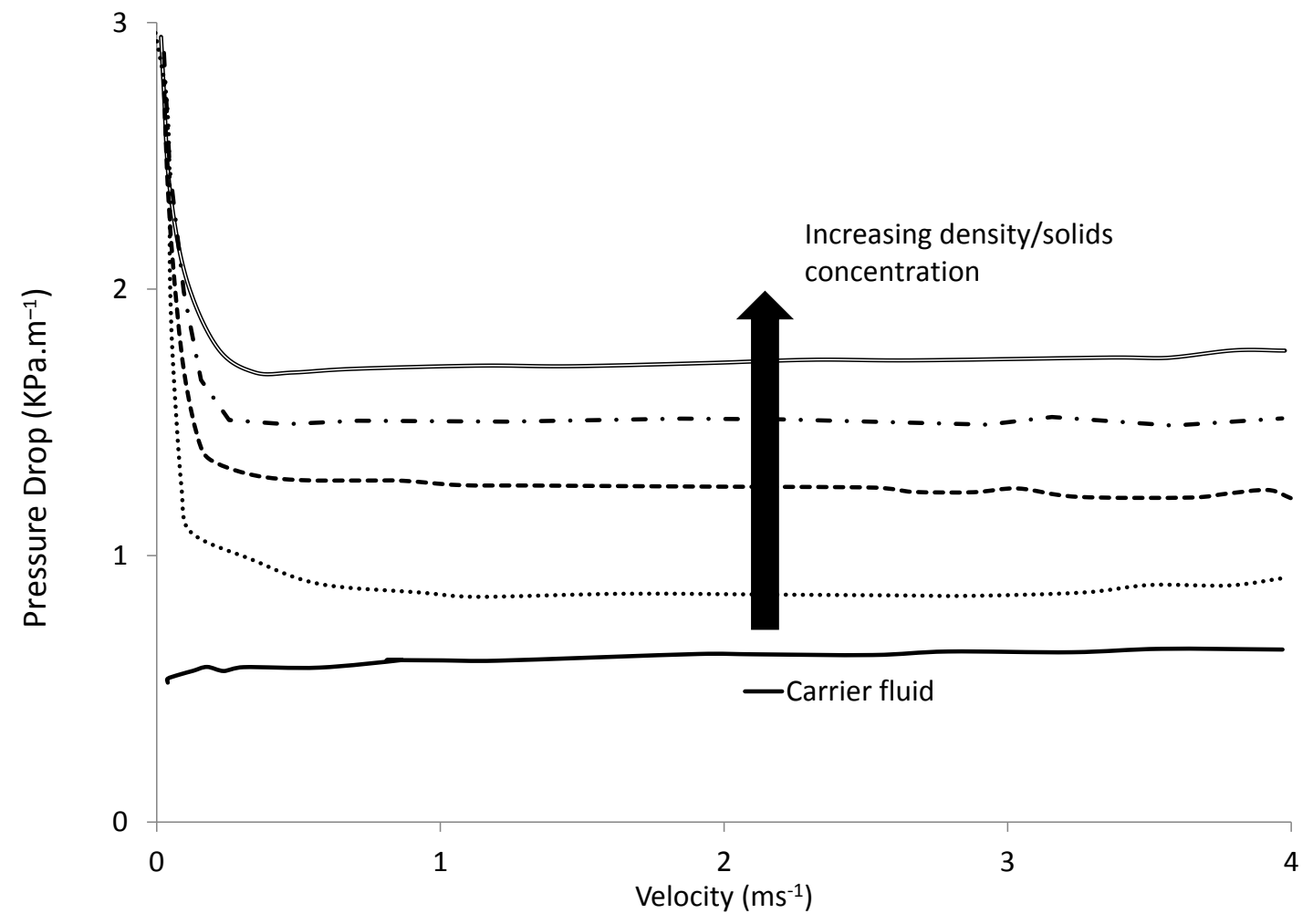

Figure 3 Pressure drop measurements on a pipeline with a sliding bed of coarse solids. Under typical operating conditions $\left({ }^{\sim} 2 \mathrm{~ms}^{-1}\right)$ there is minimal variation in measured pressure drop with changes in throughput

\section{Conclusions}

This paper has outlined the importance of taking a 'whole of process' view to tailings disposal and the requirement for a sound understanding of the interactions between the various components of a tailings circuit. Such an understanding would provide the basis for evaluating the merits of flowsheet options for tailings disposal for differing mineral systems, topography, climate, financial considerations and environmental constraints, as well as allowing the optimisation of existing tailings circuits and the testing of 'what if' scenarios.

To this end, a new comprehensive research project has been developed by CSIRO and collaborators, The University of Western Australia and ATC Williams Pty Ltd (through AMIRA International), to consider the major aspects of tailings management in an integrated approach. The project will be conducted by a multidisciplinary research team with skills and experience across all key aspects of tailings disposal. This 
project will develop an integrated set of tools for the design, management and optimisation of the tailings process to enable the implementation of leading practice and novel tailings solutions with reduced risk and uncertainty. This will be achieved by applied research in the key areas of tailings management such as water management, TSF growth and stability and the transport of tailings.

\section{Acknow l edgements}

Thanks to AngloGold Ashanti for permission given to CSIRO to outline the Sunrise Dam case study in this paper.

\section{References}

Boger, D.V. (2011) A personal perspective on paste and thickened tailings - a decade on, in Proceedings 14th International Seminar on Paste and Thickened Tailings (Paste2011), R.J. Jewell and A.B. Fourie (eds), 5-7 April 2011, Perth, Australia, Australian Centre for Geomechanics, Perth, pp. 3-11.

DITT (2007) Department of Industry Tourism and Trade, Commonwealth of Australia. Leading Practice Sustainable Development Program for the Mining Industry-Tailings Handbook.

ERCB (2009)Energy Resources Conservation Board of Alberta. Directive 074, Tailings Performance Criteria and Requirements for Oil Sands Mining Schemes, viewed 20 October 2011, www.ercb.ca/docs/documents/directives/Directive074.pdf.

Jewell, R.J. and Fourie, A.B. (eds) (2006) Paste and Thickened Tailings - A Guide, 2nd edition, Australian Centre for Geomechanics, Perth, $242 \mathrm{p}$.

Nguyen, Q.D. and Boger, D.V. (1998) Application of rheology to solving tailings disposal problems, International Journal of Mineral Processing, Vol. 54 (3), pp. 217-233.

Pullum, L. (2007) Pipelining tailings, pastes and backfill, in Proceedings Tenth International Seminar on Paste and Thickened Tailings (Paste07), A.B. Fourie and R.J. Jewell (eds), 13-15 March 2007, Perth, Australia, Australian Centre for Geomechanics, Perth, pp. 113-128.

Seddon, K.D. and Dillon, M.J. (2009) The effect of evaporation on strength and the stability of thickened tailings beach slope, in Proceedings 12th International Seminar on Paste and Thickened Tailings (Paste09), R.J. Jewell, A.B. Fourie, S. Barrera, J. Wiertz (eds), 21-24 April 2009, Viña Del Mar, Chile, Gecamin Limited, Santiago, Australian Centre for Geomechanics, Perth, pp. 261-270.

Simms, P., Williams, M.P.A., Fitton, T.G. and McPhail, G. (2011) Beaching Angles and evolution of stack geometry for thickened tailings-a review, in Proceedings 14th International Seminar on Paste and Thickened Tailings (Paste2011), R.J. Jewell and A.B. Fourie (eds), 5-7 April 2011, Perth, Australia, Australian Centre for Geomechanics, Perth, pp. 323-338.

Vietti, A., Boshoff, J.C.J. and Cope, A. (2010) Does thickening save water?, in Proceedings 13th International Seminar on Paste and Thickened Tailings (Paste2010), R.J. Jewell and A.B. Fourie (eds), 3-6 May 2010, Toronto, Canada, Australian Centre for Geomechanics, Perth, pp. 33-39. 
